\title{
Baremos del test de aptitudes mentales primarias para universitarios hondureños
}

Lina María Mendoza Recarte ${ }^{1}$

\section{RESUMEN}

La baremación de los test psicométricos es muy importante para la justa toma de decisiones. En este sentido, la OMS (2011) plantea que para la medición de los atributos intelectuales las pruebas ofrecen una guía acerca de la capacidad de la persona, pero solo se deben usar si los tests han sido tipificados con la población en la que se están aplicando. Por su parte, la American Psychological Association (APA) (1994) citado por Comisión Internacional de Test (2014), plantea la obligación de respetar los criterios de construcción de los test y todo lo relativo a su aplicación, calificación e interpretación, de tal manera que los criterios normativos son poco convenientes cuando se usan para explicar los resultados obtenidos en contextos y poblaciones diferentes a los de su creación. En consecuencia, el propósito del presente estudio fue elaborar los baremos del test de aptitudes mentales primarias (PMA) para estudiantes universitarios hondureños. La muestra se conformó con 2,588 alumnos; distribuidos por sexo en $63.3 \%$ mujeres y $36.7 \%$ hombres. La investigación se realizó con los datos recolectados durante el tercer periodo académico de 2014 y primero y segundo periodo académico de 2015, en la ciudad universitaria de Tegucigalpa, UNAH-Valle de Sula, CURLA y CURLP. Se calcularon estadísticas de tendencia central y dispersión para cada una de las escalas del PMA; posteriormente, se transformaron los puntajes brutos a datos percentiles, un baremo de la muestra general y dos que corresponden a los baremos según sexo. En los estadísticos calculados se observaron diferencias por sexo, por modalidad de educación media, por centro regional y por edad de los participantes, por lo que se compararon las puntuaciones a través de la prueba T de student y análisis de varianza. Para comparar las puntuaciones de los percentiles del baremo español y del construido en este estudio, se tomó una submuestra, de 1987, con sujetos de ambos sexos, se calculó el coefi-

\footnotetext{
${ }^{1}$ Beneficiaria de una beca básica de la DICYP, coordinadora académica de la Carrera de Psicología y profesora de la Escuela de Ciencias Psicológicas, Facultad de Ciencias Sociales, UNAH: marialina09@hotmail.com
} 
ciente de correlación de Pearson que se obtuvo de diferencias estadísticamente significativas en las cinco subpruebas del PMA.

Palabras clave: baremación, habilidades mentales primarias, universitarios.

\section{ABSTRACT}

The use of normative data regarding psychometric tests plays an important role in decision making. In this sense, the World Health Organization (2011) supports the claim that psychometric test that measure intellectual attributes, offer a guide regarding personal capacities, yet the test should only be used if it has been previously validated in the population in which it is applied. On the other hand, the American Psychological Association (APA) establishes the obligation of respecting the criteria used for constructing the test, including its application, scoring and interpretation. However, it is inconvenient to use normative test criteria when they are used to explain the results obtained in different contexts and population than the ones considered in the test construction. Therefore, the purpose of the study was to construct the normative data for Honduran university students, regarding the Primary Mental Abilities Test (PMA). The sample included 2588 students, from which $63.3 \%$ were women and $36.7 \%$ men. The data was recollected during the third academic term of 2014 as well as the first and second term of 2015, samples were taken from different campuses, which included Ciudad Universitaria, UNAH-Valle de Sula, CURLA and CURLP. Central tendency and dispersion statistics were calculated for each of the Afterwards the raw data was transformed into percentiles; normative data was created for the general population, as well as for gender. T test and ANOVA were used to evaluate the differences by gender, secondary education modality, university campus and age. In order to compare the percentile scores between the normative data constructed in this study with the Spanish dataset, a subsample consisting of 1987 subjects, from both genders, was taken into account. The scores were compared using Pearson product-moment correlation coefficient, which reflected statistical significant differences in the five PMA subtests, which indicate lower percentiles regarding the raw scores converted from the Spanish normative data.

Keywords: normative data, primary mental abilities, university students. 


\section{INTRODUCCIÓN}

En psicometría se emplean diversos instrumentos de medición para valorar varios aspectos de la conducta humana, sin embargo, es necesario contar con baremos 0 normas que conlleven a evaluaciones que le hagan justicia a una determinada población y así evitar que se comparen las ejecuciones de un grupo con las obtenidas por otros grupos que poseen abismales diferencias socioculturales y económicas.

Los test psicológicos permiten obtener objetivamente, con la mayor certeza posible, información sobre la expresión de fenómenos que suceden en la conducta humana como unidad biopsicosocial (Morales, 2009). No obstante, en Honduras, pese a su frecuente uso, la mayoría son débiles en cuanto al baremo y la actualidad de las normas, por lo que es fundamental atender esta problemática, de lo contrario se tiende a caer en errores de interpretación y predicción, pues las valoraciones se realizan con base a baremos construidos en otros países o con estandarizaciones y adaptaciones que han caído en la obsolescencia.

Al respecto, en la década de los años 80 varios psicólogos de la UNAH construyeron baremos hondureños para diferentes pruebas psicológicas (Aguilar, 1980; Barahona de Villanueva, 1981; Rivera de Donaire, 1981; Rivera, 1981; Vindel, 1985), pero, en la actualidad solo se cuenta con el trabajo de Matamoros, Moncada y Rivera (2014), quienes trabajaron en la construcción de datos normativos hondureños para el test gestáltico visomotor de Bender infantil.

Según Aliaga y Giove (1993) citado por (Grajeda Montalvo, 2010), se necesita actualizar los baremos de los test, pues los atributos psicológicos actuales tienen diferencias grandes con los de individuos de décadas pasadas, lo que conlleva a cambios en las puntuaciones típicas. Por ello, para que la estimación sea correcta, es preciso reforzar la baremación como la piedra angular para interpretar los puntajes y tomar decisiones justas basadas en los grupos de estandarización con poblaciones propias. Consecuente con la justicia, pertinencia y equidad en la que se fundamenta la UNAH, cobra especial importancia la determinación de baremos del test de aptitudes mentales primarias de Thurstone, ya que esta prueba es una de las más utilizadas en Honduras para la toma de decisiones en psicología clínica, educativa, organizacional y vocacional en adolescentes y adultos. De este modo, es obvia la inconveniencia de utilizar normas extranjeras para evaluar la población nacional.

Por tanto, es importante contar con instrumentos adecuados para la intervención 
psicológica y la investigación aplicada, ya que la adaptación de los test responde a razones de índole científico, pero también de orden práctico. El uso de los test psicométricos en la intervención psicológica requiere que se adecuen a la realidad del contexto, lo cual se logra con la estandarización del test, que es una de las mayores exigencias que deben ser cumplida a cabalidad, dado se asegura con ello que los resultados se analicen con objetividad y se ayude a la toma de decisiones con un alto índice de certidumbre.

Sin baremos locales se corre el riesgo de realizar orientaciones vocacionales erróneas, rechazar candidatos idóneos para optar a becas de estudios en determinadas áreas, imprecisiones en el diseño de planes remediales psicopedagógicos que al estar mal estructurados no conllevan al progreso académico o en la selección de personal, el rechazo de un postulante que solicita un empleo específico, etc.

Para los psicólogos de distintas áreas este estudio aporta un baremo para evaluar las aptitudes de manera justa. Con la adaptación del PMA se pretende retomar esta práctica tan importante que desde hace más de tres décadas ha sido abandonada, por lo cual dichos instrumentos, que antes fueron normalizados, han caído en la obsolescencia 0 no se tienen baremos nacionales, así que cuando se evalúa se toman decisiones sobre la base de datos normativos de otros contextos, lo que conlleva a comparar la población hondureña con un estándar ajeno a la cultura local.

\section{MÉTODO}

\section{Diseño}

La presente investigación es cuantitativa, descriptiva, transversal, no experimental de tipo correlacional. Se aplicó la onceava edición del test de aptitudes mentales primarias, PMA, para medir las habilidades cognitivas de los estudiantes de la UNAH de Tegucigalpa, UNAH-Valle de Sula, CURLA de La Ceiba y CURLP de Choluteca. Al respecto, Hernández, Fernández y Baptista (2010) explican que el enfoque cuantitativo: "Usa la recolección de datos para probar hipótesis con base en la medición numérica y el análisis estadístico para establecer patrones de comportamiento" (p.4)

Se determinaron relaciones entre las variables sexo, carrera cursada en el nivel de educación media, edad y centro regional y las aptitudes mentales primarias sin precisar ni analizar sentido de causalidad (Hernández et al, 2010). Los datos fueron reco- 
gidos durante el tercer periodo académico de 2014 y los dos primeros de 2015. Esta modalidad de estudio se diseña para medir la prevalencia de una exposición o resultado en una población definida y en un punto específico de tiempo (Hernández et al, 2010).

\section{Población}

El universo estuvo constituido por los estudiantes matriculados en la Universidad Nacional Autónoma de Honduras que hasta el II periodo de 2015 asciende a más de 75,000 alumnos; de los cuales la mayor concentración poblacional está en la ciudad universitaria de Tegucigalpa con 48,854 discentes. En la UNAH- Valle de Sula asisten más de 15,477 alumnos; en el CURLA la población supera los 1,496 y en el CURLP está por arriba de los 15,41personas (DIPP, 2015).

La población estudiantil de la UNAH es pertinente y se considera lo suficientemente representativa para la baremación del test PMA, en vista de que a la máxima casa de estudios concurren personas de diferentes centros educativos del nivel secundario del país, además de ser la institución que alberga la mayor cantidad de estudiantes matriculados en el nivel superior hondureño, procedentes de todos los departamentos de Honduras.

La muestra la constituyeron 2,588 estudiantes (hombres y mujeres) de primer ingreso y de reingreso de diferentes niveles académicos matriculados en la UNAH en el III periodo académico de 2014 y I y II periodos académicos de 2015; se conformó con sujetos pertenecientes a ciudad universitaria de Tegucigalpa, UNAH-VS, CURLA y CURLP, en vista de que estos centros son los de mayor antigüedad y por tradición a ellos confluyen personas de los cuatro puntos cardinales del país, de esta manera se logró en la muestra la representación de los 18 departamentos de Honduras.

El muestreo utilizado fue de tipo probabilístico estratificado, lo que permite aumentar la precisión de la muestra para cada estrato (Hernández et al, 2010). Se procedió a calcular el tamaño de la muestra, la estratificación y afijación muestral por facultad/centro regional y carrera; luego se seleccionaron las unidades (secciones) en las que se aplicó el test con base al número de matriculados y las secciones establecidas, considerando las de mayor número de estudiantes. Se descartaron las carreras con pocos estudiantes y el número de la muestra que le correspondía se distribuyó entre las secciones seleccionadas. 


\section{Entorno}

Todo el proceso se realizó en la ciudad universitaria de Tegucigalpa y los centros regionales de La Ceiba (CURLA), San Pedro Sula (UNAH-VS) y Choluteca (CURLP). La UNAH es una institución educativa de nivel superior, fue fundada por el presbítero José Trinidad Reyes el 19 de septiembre de 1847 y desde entonces ha sido la máxima casa de estudios del nivel universitario. Cuenta con un campus central en Tegucigalpa, ocho centros regionales, ocho centros de educación a distancia y cuatro telecentros distribuidos por todo el territorio nacional.

\section{Intervenciones}

Se utilizó como instrumento de medición la onceava edición (tipificación española) del test de aptitudes mentales primarias de Thurstone, editado por el Departamento I+D de TEA Ediciones. El instrumento fue aplicado de forma colectiva, con una duración de 60 minutos aproximadamente; de los cuales 26 minutos son de trabajo efectivo, el resto corresponde al rapport y a las instrucciones.

Para la recolección de datos se aplicó la prueba siguiendo al pie de la letra el procedimiento de administración especificado en el manual y contando con los requerimientos generales para la administración de test psicológicos. Posteriormente, se procedió al escrutinio de cada instrumento para obtener las puntuaciones directas por cada uno de los casos en cada subtest y con ello se construyó una matriz de datos crudos en Excel 2013, con los que se realizó el tratamiento estadístico utilizando el SPSS 20.

\section{Análisis estadístico}

Para el procesamiento y análisis de los datos obtenidos mediante la aplicación del test de aptitudes mentales primarias se emplearon los programas estadísticos SPSS 20 y Excel 2013. Para mostrar concordancia con los objetivos planteados, se utilizaron estadísticos descriptivos y de frecuencia con los que se han hecho los resúmenes numéricos del conjunto de datos (Coolican, 2005), que de acuerdo con Downie y Heath (1986) proporcionan información y describen la muestra que se estudia. Igualmente, se eligieron las medidas de tendencia central (media, mediana y moda) y las de dispersión (desviación típica, máximo y mínimo).

Se calculó la media, mediana, moda, varianza, desviación estándar, coeficiente de Curtosis y la correlación -como otra medida descriptiva- para determinar la relación 
entre las variables de estudio. No obstante, para establecer la significancia de las correlaciones se recurrió al empleo de estadísticos inferenciales.

En el análisis de la confiabilidad se calculó el error estándar de medición, ya que resulta inconveniente aplicar procedimientos estadísticos de fórmula par-impar, dada la naturaleza de los subtest en los que se requiere velocidad para la resolución de los ítems. Al respecto, Casullo y Echeverría (2010) explican que cuando se trata de estimar la confiabilidad de resultados en los que se determinan restricciones en cuanto al tiempo concedido para cada subtest, es inapropiado el uso de estas formas de cálculo de confiabilidad.

\section{RESULTADOS}

La muestra fue de 2,588 estudiantes, calculada con un nivel de confianza del $95 \%$ y un margen de error del $5 \%$. Eran 1,637 mujeres, es decir, el $63.3 \%$ y 951 varones, lo que representa el $36.7 \%$ (ver tabla 1). Los sujetos tenían entre 15 y 59 años, con una media total de 22 años; de ellos, 1,540 oscilaban entre los 18 y 22 años (59.5\%) y solo el $1.5 \%$ (39 personas) se encontraba entre las edades de más de 37 años.

Se considera que la población estudiantil de la UNAH es pertinente y lo suficientemente representativa para los efectos de normalización del test en cuestión en vista de que a ella concurren personas de diferentes centros educativos del país además de ser la institución que alberga la mayor cantidad de estudiantes del nivel superior hondureño. Se suma el hecho de que la muestra está constituida por participantes del total de los dieciocho departamentos de Honduras.

Los cuatro centros universitarios fueron seleccionados de acuerdo a la cantidad de estudiantes inscritos y de conformidad al presupuesto asignado. Corresponde a Tegucigalpa el $63.7 \%$ de los sujetos, el $16.8 \%$ eran de UNAH-Valle de Sula, el 11.3 $\%$ del CURLA y el $7.7 \%$ del CURLP. La muestra incluye participantes de los 18 departamentos de Honduras, en su mayoría son de los departamentos de Francisco Morazán y Cortés, con el $36.2 \%$ y un $12.2 \%$, respectivamente. Menos del $1 \%$ procede de los departamentos de Copán, Gracias a Dios, Intibucá, Islas de la Bahía, La Paz, Lempira, Ocotepeque, Santa Bárbara y Valle. 
Tabla 1. Sexo y media de edad de los participantes

\begin{tabular}{|l|lll|}
\hline Sexo & $\boldsymbol{N}$ & $\%$ & Media de edad \\
\hline Masculino & 951 & 36.7 & 21.85 \\
Femenino & 1637 & 63.3 & 22.14 \\
Total & 2588 & 100 & 22.03 \\
\hline
\end{tabular}

Fuente: Elaboración propia

De la muestra, el $26.2 \%$ eran de carreras de la Facultad de Ciencias Sociales; el 16.1 $\%$ de la Facultad de Ciencias Económicas; el $12.9 \%$ de la Facultad de Humanidades y Artes y el $10.2 \%$ de Ciencias Médicas. Entre el 7 y $3 \%$ estudiaban carreras de las facultades de Ciencias, Odontología, Ingeniería, Ciencias Agrícolas y Ambientales, Derecho y Química y Farmacia. Menos del 1 \% pertenecían a la Facultad de Ciencias Espaciales.

Según el nivel de educación media, corresponden al bachillerato científico humanista un $30.6 \%$, a los bachilleratos administrativos y comercio el $20.4 \%$ y a los bachilleratos técnicos el $10.5 \%$.

De ese modo, antes de proceder a la construcción de los baremos hondureños del PMA, se procedió a calcular el error estándar de medición, a fin de evaluar la confiabilidad de las puntuaciones directas de los subtests, éste representa una estimación estadística del grado en que el puntaje bruto obtenido por un participante se acerca a su puntaje "verdadero" teórico (Casullo y Echeverría, 2010; Morales, 2007 y Rivera y Camoriano, 1981).

En la tabla 2 se observa que el error estándar de medición por sexo es muy cercano a cero. Siendo el número de sujetos $(\mathrm{N})$ relativamente grande, esto indica que la media de la muestra es muy cercana a la media de la población.

Por otra parte, con las puntuaciones naturales se procedió a la elaboración del baremo percentilar de la muestra general. Se construyeron los datos normativos en cada una de las cinco subpruebas del PMA, los cuales se presentan en la siguiente Tabla No. 3. 
Tabla 2. Error estándar de medición

\begin{tabular}{|c|c|c|c|c|c|c|c|c|c|c|}
\hline \multicolumn{6}{|c|}{ Mujeres } & \multicolumn{5}{|c|}{ Varones } \\
\hline & $\begin{array}{l}\overline{\widetilde{\sigma}} \\
\text { 遃 }\end{array}$ & $\begin{array}{l}\overline{\bar{\sigma}} \\
\overline{\mathrm{d}} \\
\overline{\mathrm{D}} \\
\overline{\mathrm{D}} \\
\end{array}$ & 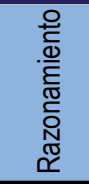 & 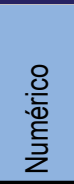 & 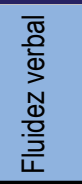 & $\begin{array}{l}\overline{\widetilde{\sigma}} \\
\stackrel{\stackrel{0}{\omega}}{2}\end{array}$ & $\begin{array}{l}\overline{\bar{\sigma}} \\
\frac{\mathrm{d}}{\mathrm{d}} \\
\overline{\mathrm{D}} \\
\mathrm{W}\end{array}$ & 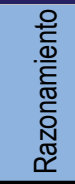 & 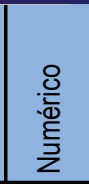 & 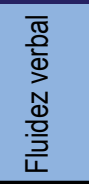 \\
\hline $\mathrm{N}$ & 1638 & 1639 & 1639 & 1639 & 1632 & 949 & 948 & 949 & 949 & 941 \\
\hline Media & 19 & 12.82 & 10.12 & 10.9 & 37.53 & 18.47 & 17.88 & 9.83 & 11.47 & 38.16 \\
\hline $\begin{array}{l}\text { Error } \\
\text { típico }\end{array}$ & .165 & .306 & .232 & . 155 & .294 & .213 & .408 & .275 & .224 & .386 \\
\hline
\end{tabular}

Fuente: Elaboración propia

Tabla 3. Datos normativos de la muestra general

\begin{tabular}{|c|c|c|c|c|c|}
\hline \multirow[t]{2}{*}{ Percentil } & \multicolumn{5}{|c|}{ Puntuaciones Naturales } \\
\hline & $\begin{array}{l}\text { Factor } \\
\text { Verbal }\end{array}$ & $\begin{array}{c}\text { Factor } \\
\text { Espacial }\end{array}$ & $\begin{array}{c}\text { Factor } \\
\text { Razonamiento }\end{array}$ & $\begin{array}{c}\text { Factor } \\
\text { Numérico }\end{array}$ & $\begin{array}{c}\text { Factor Fluidez } \\
\text { Verbal }\end{array}$ \\
\hline 1 & $0-2$ & - & 0 & - & $0-7$ \\
\hline 2 & $3-5$ & - & - & - & $8-12$ \\
\hline 3 & 6 & - & - & - & $13-16$ \\
\hline 4 & 7 & - & 1 & - & 17 \\
\hline 5 & 8 & - & - & 0 & $18-19$ \\
\hline 6 & - & - & 2 & - & 20 \\
\hline 7 & 9 & - & - & - & 21 \\
\hline 8 & - & - & & 1 & 22 \\
\hline 9 & - & - & - & - & 23 \\
\hline 10 & 10 & - & - & 2 & \\
\hline
\end{tabular}




\begin{tabular}{|c|c|c|c|c|c|}
\hline \multirow[t]{2}{*}{ Percentil } & \multicolumn{5}{|c|}{ Puntuaciones Naturales } \\
\hline & $\begin{array}{l}\text { Factor } \\
\text { Verbal }\end{array}$ & $\begin{array}{c}\text { Factor } \\
\text { Espacial }\end{array}$ & $\begin{array}{c}\text { Factor } \\
\text { Razonamiento }\end{array}$ & $\begin{array}{c}\text { Factor } \\
\text { Numérico }\end{array}$ & $\begin{array}{c}\text { Factor Fluidez } \\
\text { Verbal }\end{array}$ \\
\hline 11 & - & - & - & - & 24 \\
\hline 12 & 11 & _ & - & - & - \\
\hline 13 & - & - & - & - & - \\
\hline 14 & - & - & - & 3 & 25 \\
\hline 15 & - & _ & - & - & - \\
\hline 16 & 12 & _- & - & - & 26 \\
\hline 17 & - & - & - & - & - \\
\hline 18 & - & _- & 3 & 4 & 27 \\
\hline 19 & - & _ & - & - & - \\
\hline 20 & - & - & - & - & - \\
\hline 21 & - & _- & & & \\
\hline 22 & - & - & - & 5 & 28 \\
\hline 23 & 13 & $0-1$ & - & - & - \\
\hline 24 & - & - & 4 & - & 29 \\
\hline 25 & - & - & - & - & \\
\hline 26 & - & 2 & - & - & 30 \\
\hline 27 & - & - & - & 6 & - \\
\hline 28 & 14 & 3 & - & - & - \\
\hline 29 & - & 4 & - & - & 31 \\
\hline 30 & - & - & - & - & - \\
\hline 31 & - & 5 & - & 7 & - \\
\hline
\end{tabular}




\begin{tabular}{|c|c|c|c|c|c|}
\hline \multirow[t]{2}{*}{ Percentil } & \multicolumn{5}{|c|}{ Puntuaciones Naturales } \\
\hline & $\begin{array}{l}\text { Factor } \\
\text { Verbal }\end{array}$ & $\begin{array}{c}\text { Factor } \\
\text { Espacial }\end{array}$ & $\begin{array}{c}\text { Factor } \\
\text { Razonamiento }\end{array}$ & $\begin{array}{c}\text { Factor } \\
\text { Numérico }\end{array}$ & $\begin{array}{c}\text { Factor Fluidez } \\
\text { Verbal }\end{array}$ \\
\hline 32 & - & - & - & - & - \\
\hline 33 & - & 6 & 5 & - & 32 \\
\hline 34 & 15 & - & - & - & - \\
\hline 35 & - & 7 & - & - & - \\
\hline 36 & - & - & - & - & - \\
\hline 37 & - & - & - & - & 33 \\
\hline 38 & - & 8 & - & 8 & - \\
\hline 39 & - & - & - & - & - \\
\hline 40 & - & 9 & 6 & - & 34 \\
\hline 41 & 16 & - & - & - & - \\
\hline 42 & - & 10 & - & - & - \\
\hline 43 & - & - & - & 9 & - \\
\hline 44 & - & - & - & - & 35 \\
\hline 45 & - & 11 & - & - & - \\
\hline 46 & - & - & 7 & - & - \\
\hline 47 & 17 & - & - & - & - \\
\hline 48 & - & 12 & - & - & - \\
\hline 49 & - & - & - & - & 36 \\
\hline 50 & - & - & - & 10 & - \\
\hline 51 & - & 13 & - & - & - \\
\hline 52 & - & - & - & - & 37 \\
\hline
\end{tabular}




\begin{tabular}{|c|c|c|c|c|c|}
\hline \multirow[t]{2}{*}{ Percentil } & \multicolumn{5}{|c|}{ Puntuaciones Naturales } \\
\hline & $\begin{array}{l}\text { Factor } \\
\text { Verbal }\end{array}$ & $\begin{array}{c}\text { Factor } \\
\text { Espacial }\end{array}$ & $\begin{array}{c}\text { Factor } \\
\text { Razonamiento }\end{array}$ & $\begin{array}{c}\text { Factor } \\
\text { Numérico }\end{array}$ & $\begin{array}{c}\text { Factor Fluidez } \\
\text { Verbal }\end{array}$ \\
\hline 53 & - & 14 & 8 & - & - \\
\hline 54 & 18 & - & - & - & - \\
\hline 55 & - & - & - & - & 38 \\
\hline 56 & - & - & - & - & - \\
\hline 57 & - & 15 & - & - & - \\
\hline 58 & - & - & - & 11 & - \\
\hline 59 & 19 & 16 & - & - & 39 \\
\hline 60 & - & - & 9 & - & - \\
\hline 61 & - & - & - & - & - \\
\hline 62 & - & 17 & - & - & - \\
\hline 63 & - & - & - & 12 & 40 \\
\hline 64 & - & - & - & - & - \\
\hline 65 & 20 & 18 & - & - & - \\
\hline 66 & - & - & - & - & 41 \\
\hline 67 & - & - & 10 & - & - \\
\hline 68 & - & 19 & - & - & - \\
\hline 69 & - & - & - & - & 42 \\
\hline 70 & 21 & - & - & 13 & - \\
\hline 71 & - & 20 & - & - & - \\
\hline 72 & - & - & 11 & & 43 \\
\hline 73 & - & 21 & - & - & - \\
\hline
\end{tabular}




\begin{tabular}{|c|c|c|c|c|c|}
\hline \multirow[t]{2}{*}{ Percentil } & \multicolumn{5}{|c|}{ Puntuaciones Naturales } \\
\hline & $\begin{array}{l}\text { Factor } \\
\text { Verbal }\end{array}$ & $\begin{array}{c}\text { Factor } \\
\text { Espacial }\end{array}$ & $\begin{array}{c}\text { Factor } \\
\text { Razonamiento }\end{array}$ & $\begin{array}{c}\text { Factor } \\
\text { Numérico }\end{array}$ & $\begin{array}{c}\text { Factor Fluidez } \\
\text { Verbal }\end{array}$ \\
\hline 74 & - & - & - & - & - \\
\hline 75 & 22 & 22 & - & - & - \\
\hline 76 & - & & 12 & 14 & 44 \\
\hline 77 & - & - & - & - & - \\
\hline 78 & - & 23 & - & - & 45 \\
\hline 79 & - & - & - & - & - \\
\hline 80 & - & 24 & - & - & 46 \\
\hline 81 & 23 & - & 13 & 15 & - \\
\hline 82 & - & - & - & - & 47 \\
\hline 83 & - & 25 & - & - & - \\
\hline 84 & - & 26 & - & 16 & 48 \\
\hline 85 & 24 & - & 14 & - & - \\
\hline 86 & - & 27 & - & - & 49 \\
\hline 87 & - & - & - & - & - \\
\hline 88 & 25 & 28 & 15 & 17 & 50 \\
\hline 89 & - & - & 16 & - & 51 \\
\hline 90 & - & 29 & & 18 & \\
\hline 91 & 26 & 30 & - & - & 52 \\
\hline 92 & 27 & 31 & 17 & & 53 \\
\hline 93 & - & 32 & - & 19 & - \\
\hline 94 & 28 & 33 & 18 & 24 & $54-55$ \\
\hline
\end{tabular}




\begin{tabular}{|r|c|c|c|c|c|}
\hline Percentil & \multicolumn{3}{|l}{ Puntuaciones Naturales } \\
\cline { 2 - 3 } & $\begin{array}{c}\text { Factor } \\
\text { Verbal }\end{array}$ & $\begin{array}{c}\text { Factor } \\
\text { Espacial }\end{array}$ & $\begin{array}{c}\text { Factor } \\
\text { Razonamiento }\end{array}$ & $\begin{array}{c}\text { Factor } \\
\text { Numérico }\end{array}$ & $\begin{array}{c}\text { Factor Fluidez } \\
\text { Verbal }\end{array}$ \\
\hline 95 & - & 34 & 19 & & 56 \\
\hline 96 & 29 & $35-37$ & 20 & $21-22$ & 57 \\
\hline 97 & 30 a 31 & $38-40$ & $21-24$ & 23 & $58-60$ \\
\hline 98 & 32 a 34 & 41 a 43 & $25-26$ & $24-26$ & $61-62$ \\
\hline 99 & 35 a 50 & 44 a 54 & $27-30$ & $27-70$ & $63-75$ \\
\hline N & 2587 & 2588 & 2588 & 2588 & 2574 \\
\hline Media & 53.89 & 40.43 & 53.9 & 61.6 & 32.6 \\
\hline DE & 27.9 & 25.1 & 27.04 & 28.2 & 27.8 \\
\hline
\end{tabular}

Fuente: Elaboración propia

Con los datos estandarizados de la muestra general se aplicaron estadísticos para determinar la existencia o no de diferencias significativas de acuerdo al sexo. La tabla 4 indica los percentiles medios obtenidos para la muestra de acuerdo al sexo de los participantes para cada una de las subpruebas del PMA.

\section{Tabla 4. Estadísticos para la diferencia de medias por sexo}

\begin{tabular}{|l|l|c|c|c|c|}
\hline $\begin{array}{c}\text { Subtest del test } \\
\text { de aptitudes mentales } \\
\text { primarias PMA }\end{array}$ & $\begin{array}{c}\text { Sexo de los } \\
\text { sujetos }\end{array}$ & $\mathbf{N}$ & Media & $\begin{array}{c}\text { Desviación } \\
\text { típica }\end{array}$ & $\begin{array}{c}\text { Error típico } \\
\text { de la media }\end{array}$ \\
\hline \multirow{2}{*}{ Compresión verbal } & Femenino & 1209 & 55.63 & 27.828 & .800 \\
\cline { 2 - 6 } & Masculino & 777 & 51.51 & 27.853 & .999 \\
\hline \multirow{2}{*}{ Concepción espacial } & Femenino & 1210 & 49.12 & 23.715 & .682 \\
\cline { 2 - 6 } & Masculino & 777 & 61.29 & 25.588 & .918 \\
\hline \multirow{2}{*}{ Razonamiento } & Femenino & 1210 & 53.59 & 26.796 & .770 \\
\cline { 2 - 6 } & Masculino & 777 & 53.92 & 27.446 & .985 \\
\hline \multirow{2}{*}{ Comprensión numérica } & Femenino & 1206 & 53.76 & 27.703 & .798 \\
\cline { 2 - 6 } & Masculino & 776 & 56.80 & 28.918 & 1.038 \\
\hline \multirow{2}{*}{ Fluidez verbal } & Femenino & 1198 & 54.02 & 27.480 & .794 \\
\cline { 2 - 6 } & Masculino & 769 & 52.46 & 28.514 & 1.028 \\
\hline
\end{tabular}

Fuente: Elaboración propia 
A continuación en la tabla No. 5 se presenta los resultados del contraste de las medias de acuerdo al sexo de los participantes.Se observan las diferencias significativas a un nivel de confianza de 95\% en los factores de Comprensión Verbal, Espacial y Comprensión Numérica.

Tabla 5. Contraste de medias de cada subtest por sexo

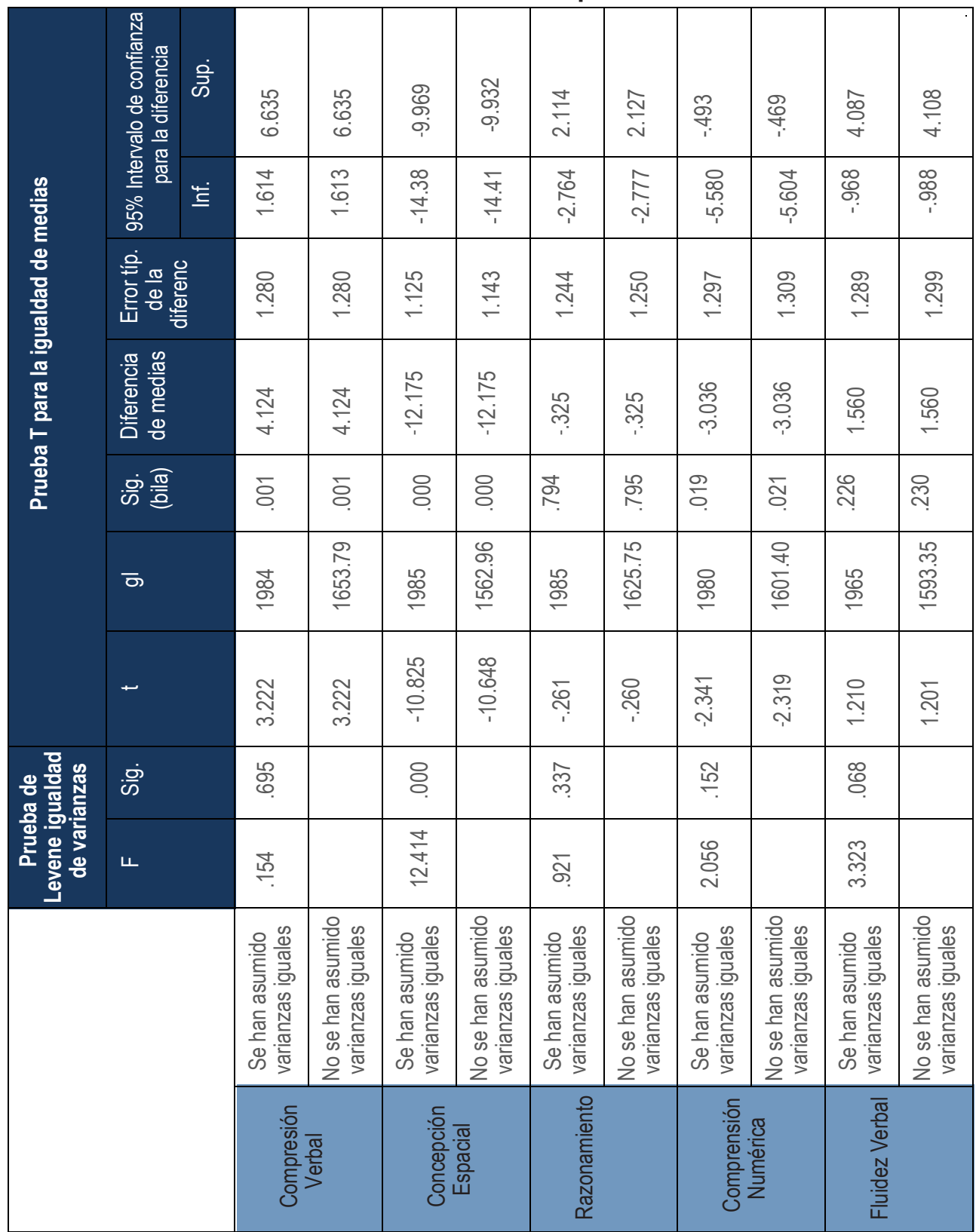

Fuente: Elaboración propia 
También se analizó si existían diferencias significativas de acuerdo a la carrera del nivel medio que cursaron los sujetos del estudio. La tabla 6 muestra que la media de calificaciones del test en tres habilidades mentales, de acuerdo a la carrera del nivel de educación media, es diferente con excepción de la concepción espacial y fluidez verbal.

Tabla 6. Análisis de varianza de baremos de acuerdo a la modalidad de nivel de educación media

\begin{tabular}{|c|c|c|c|c|c|c|}
\hline \multicolumn{2}{|c|}{ Subtest del PMA } & Suma de & gl & Media & $\mathbf{F}$ & Sig. \\
\hline \multirow{3}{*}{$\begin{array}{l}\text { Compresión } \\
\text { verbal }\end{array}$} & Intergrupos & 22238.549 & 2 & 11119.275 & 14.590 & .000 \\
\hline & Intragrupos & 1481567.9 & 1944 & 762.123 & & \\
\hline & Total & 1503806.5 & 1946 & & & \\
\hline \multirow{3}{*}{$\begin{array}{l}\text { Concepción } \\
\text { espacial }\end{array}$} & Intergrupos & 2847.892 & 2 & 1423.946 & 2.252 & .105 \\
\hline & Intragrupos & 1229762.9 & 1945 & 632.269 & & \\
\hline & Total & 1232610.8 & 1947 & & & \\
\hline \multirow[t]{3}{*}{ Razonamiento } & Intergrupos & 6733.909 & 2 & 3366.954 & 4.632 & .010 \\
\hline & Intragrupos & 1413749.7 & 1945 & 726.864 & & \\
\hline & Total & 1420483.6 & 1947 & & & \\
\hline \multirow{3}{*}{$\begin{array}{l}\text { Comprensión } \\
\text { numérica }\end{array}$} & Intergrupos & 8414.976 & 2 & 4207.488 & 5.330 & .005 \\
\hline & Intragrupos & 1531519.5 & 1940 & 789.443 & & \\
\hline & Total & 1539934.5 & 1942 & & & \\
\hline \multirow[t]{3}{*}{ Fluidez verbal } & Intergrupos & 519.135 & 2 & 259.568 & .332 & .718 \\
\hline & Intragrupos & 1506352.6 & 1925 & 782.521 & & \\
\hline & Total & 1506871.7 & 1927 & & & \\
\hline
\end{tabular}

Fuente: Elaboración propia 
Con relación a la edad de los sujetos del estudio, en la tabla 7 se observa las diferencias en los factores espacial, numérico y razonamiento, en tanto que el verbal y el de fluidez verbal no son diferentes.

Tabla 7. Análisis de la varianza para los baremos hondureños de acuerdo a la edad de los participantes

\begin{tabular}{|c|c|c|c|c|c|c|}
\hline & & $\begin{array}{l}\text { Suma de } \\
\text { cuadrados }\end{array}$ & gl & $\begin{array}{c}\text { Media } \\
\text { cuadrática }\end{array}$ & F & Sig. \\
\hline \multirow{3}{*}{$\begin{array}{l}\text { Compresión } \\
\text { verbal }\end{array}$} & Intergrupos & 24770.217 & 22 & 1125.919 & 1.453 & .080 \\
\hline & Intragrupos & 1520811.056 & 1963 & 774.738 & & \\
\hline & Total & 1545581.273 & 1985 & & & \\
\hline \multirow{3}{*}{$\begin{array}{l}\text { Concepción } \\
\text { espacial }\end{array}$} & Intergrupos & 29149.621 & 22 & 1324.983 & 2.117 & .002 \\
\hline & Intragrupos & 1229011.111 & 1964 & 625.769 & & \\
\hline & Total & 1258160.732 & 1986 & & & \\
\hline \multirow[t]{3}{*}{ Razonamiento } & Intergrupos & 53770.267 & 22 & 2444.103 & 3.431 & .000 \\
\hline & Intragrupos & 1398941.470 & 1964 & 712.292 & & \\
\hline & Total & 1452711.737 & 1986 & & & \\
\hline \multirow{3}{*}{$\begin{array}{l}\text { Comprensión } \\
\text { numérica }\end{array}$} & Intergrupos & 34563.817 & 22 & 1571.083 & 1.995 & .004 \\
\hline & Intragrupos & 1542632.726 & 1959 & 787.459 & & \\
\hline & Total & 1577196.543 & 1981 & & & \\
\hline \multirow[t]{3}{*}{ Fluidez verbal } & Inter-grupos & 25674.924 & 22 & 1167.042 & 1.509 & .061 \\
\hline & Intra-grupos & 1503792.989 & 1944 & 773.556 & & \\
\hline & Total & 1529467.913 & 1966 & & & \\
\hline
\end{tabular}

Fuente: Elaboración propia 
También se compararon los datos normativos por centro regional. Los resultados de la tabla 8 demuestran que en todos los subtest que mide el PMA se observan diferencias significativas.

Tabla 8. Comparación de los datos normativos hondureños de acuerdo al centro regional universitario

\begin{tabular}{|c|c|c|c|c|c|c|}
\hline & & $\begin{array}{l}\text { Suma de } \\
\text { cuadrados }\end{array}$ & gl & $\begin{array}{c}\text { Media } \\
\text { cuadrática }\end{array}$ & $F$ & Sig. \\
\hline \multirow{3}{*}{$\begin{array}{l}\text { Compresión } \\
\text { verbal }\end{array}$} & Intergrupos & 9215.925 & 3 & 3071.975 & 3.963 & .008 \\
\hline & Intragrupos & 1536365.347 & 1982 & 775.159 & & \\
\hline & Total & 1545581.273 & 1985 & & & \\
\hline \multirow{3}{*}{$\begin{array}{l}\text { Concepción } \\
\text { espacial }\end{array}$} & Intergrupos & 10137.461 & 3 & 3379.154 & 5.369 & .001 \\
\hline & Intragrupos & 1248023.271 & 1983 & 629.361 & & \\
\hline & Total & 1258160.732 & 1986 & & & \\
\hline \multirow[t]{3}{*}{ Razonamiento } & Intergrupos & 15866.087 & 3 & 5288.696 & 7.299 & .000 \\
\hline & Intragrupos & 1436845.651 & 1983 & 724.582 & & \\
\hline & Total & 1452711.737 & 1986 & & & \\
\hline \multirow{3}{*}{$\begin{array}{l}\text { Comprensión } \\
\text { numérica }\end{array}$} & Intergrupos & 51158.786 & 3 & 17052.929 & 22.103 & .000 \\
\hline & Intragrupos & 1526037.757 & 1978 & 771.505 & & \\
\hline & Total & 1577196.543 & 1981 & & & \\
\hline \multirow[t]{3}{*}{ Fluidez verbal } & Intergrupos & 27432.363 & 3 & 9144.121 & 11.950 & .000 \\
\hline & Intragrupos & 1502035.550 & 1963 & 765.173 & & \\
\hline & Total & 1529467.913 & 1966 & & & \\
\hline
\end{tabular}

Fuente: Elaboración propia

Se compararon los datos normativos españoles y hondureños obtenidos por una submuestra de 1,987 sujetos. La tabla 9 muestra las medias y desviaciones estándar de los datos percentilares. 
Se compararon los datos normativos españoles y hondureños obtenidos por una submuestra de 1,987 sujetos. La tabla 9 muestra las medias y desviaciones estándar de los datos percentilares.

Tabla 9. Medias y desviaciones estándar de los baremos españoles y hondureños

\begin{tabular}{|c|c|c|c|c|}
\hline \multirow[t]{2}{*}{ Sub test } & \multicolumn{2}{|l|}{ Medias } & \multicolumn{2}{|c|}{ Desviación típica } \\
\hline & Español & Hondureño & Español & Hondureño \\
\hline Factor V & 9.36 & 54.02 & 8.838 & 27.904 \\
\hline Factor E & 28.91 & 53.88 & 27.598 & 25.17 \\
\hline Factor $\mathbf{R}$ & 35.07 & 53.72 & 26.872 & 26.046 \\
\hline Factor $\mathrm{N}$ & 4.69 & 54.95 & 7.564 & 28.216 \\
\hline Factor F & 23.95 & 53.41 & 22.167 & 27.892 \\
\hline
\end{tabular}

Fuente: Elaboración propia

También se calculó la correlación de las puntuaciones mediante el coeficiente producto-momento de Pearson. La tabla 10 muestra cuatro coeficientes fuertes y uno moderado estadísticamente significativos.

Tabla 10. Correlaciones de los percentiles obtenidos por la muestra de acuerdo a los baremos hondureños y españoles

\begin{tabular}{|c|c|c|c|c|c|c|}
\hline \multicolumn{2}{|c|}{$\begin{array}{l}\text { Baremo español } \\
\text { Baremo hondureño }\end{array}$} & Factor V & Factor $E$ & Factor $\mathbf{R}$ & Factor $\mathbf{N}$ & Factor F \\
\hline \multirow{3}{*}{ Factor V } & $\mathrm{r}$ & $.742^{* *}$ & & & & \\
\hline & Sig. (bilat.) & .000 & & & & \\
\hline & $\mathrm{n}$ & 1974 & & & & \\
\hline \multirow{3}{*}{ Factor $E$} & $\mathrm{r}$ & & $.943^{\star *}$ & & & \\
\hline & Sig. (bilat.) & & .000 & & & \\
\hline & $\mathrm{n}$ & & 1975 & & & \\
\hline \multirow{3}{*}{ Factor $\mathbf{R}$} & $\mathrm{r}$ & & & $.908^{* \star}$ & & \\
\hline & Sig. (bilat.) & & & .000 & & \\
\hline & $\mathrm{n}$ & & & 1973 & & \\
\hline \multirow{3}{*}{ Factor $\mathbf{N}$} & $\mathrm{r}$ & & & & $.485^{\star *}$ & \\
\hline & Sig. (bilat.) & & & & .000 & \\
\hline & $\mathrm{n}$ & & & & 1975 & \\
\hline \multirow{3}{*}{ Factor $F$} & $\mathrm{r}$ & & & & & $.858^{* *}$ \\
\hline & Sig. (bilat.) & & & & & .000 \\
\hline & $\mathrm{n}$ & & & & & 1963 \\
\hline & \multicolumn{6}{|c|}{ ** La correlación es significativa al nivel 0,01 (bilateral). } \\
\hline
\end{tabular}

Fuente: Elaboración propia 
Una vez realizados los estadísticos presentados anteriormente, en atención a los objetivos de este estudio, a continuación se presentan las tablas que corresponden a los baremos de la muestra general de acuerdo al sexo.

\section{Tabla 11. Baremo Percentilar Femenino}

\begin{tabular}{|c|c|c|c|c|c|}
\hline \multicolumn{2}{|c|}{ FACTOR VERBAL } & \multicolumn{2}{|c|}{ FACTOR ESPACIAL } & \multicolumn{2}{|c|}{ FACTOR RAZONAMIENTO } \\
\hline Percentil & Puntuación natural & Percentil & Puntuación natural & Percentil & Puntuación natural \\
\hline 1 & 0 a 2 & 27 & 0 a 1 & 1 & 0 \\
\hline 2 & 3 a 5 & 29 & 2 & 3 & 1 \\
\hline 3 & 6 & 32 & 3 & 9 & 2 \\
\hline 4 & 7 & 33 & 4 & 18 & 3 \\
\hline 6 & 8 & 36 & 5 & 27 & 4 \\
\hline 7 & 9 & 38 & 6 & 33 & 5 \\
\hline 11 & 10 & 41 & 7 & 39 & 6 \\
\hline 13 & 11 & 44 & 8 & 46 & 7 \\
\hline 16 & 12 & 46 & 9 & 53 & 8 \\
\hline 23 & 13 & 49 & 10 & 60 & 9 \\
\hline 26 & 14 & 51 & 11 & 67 & 10 \\
\hline 33 & 15 & 55 & 12 & 72 & 11 \\
\hline 39 & 16 & 59 & 13 & 76 & 12 \\
\hline 45 & 17 & 62 & 14 & 80 & 13 \\
\hline 52 & 18 & 64 & 15 & 84 & 14 \\
\hline 57 & 19 & 67 & 16 & 88 & 15 \\
\hline 63 & 20 & 70 & 17 & 89 & 16 \\
\hline 68 & 21 & 73 & 18 & 92 & 17 \\
\hline 74 & 22 & 75 & 19 & 94 & 18 \\
\hline 80 & 23 & 78 & 20 & 95 & 19 \\
\hline 84 & 24 & 80 & 21 & 96 & 20 \\
\hline 87 & 25 & 81 & 22 & 97 & 21 a 24 \\
\hline 90 & 26 & 84 & 23 & 98 & 25 a 29 \\
\hline 91 & 27 & 85 & 24 & 99 & 30 \\
\hline 94 & 28 & 88 & 25 a 26 & & \\
\hline 96 & $29-30$ & 90 & 27 & & \\
\hline 97 & 31 a 32 & 92 & 28 & & \\
\hline 98 & 33 a 34 & 93 & 29 & & \\
\hline 99 & $35-50$ & 94 & 30 & & \\
\hline & & 95 & 31 a 32 & & \\
\hline & & 96 & 33 a 34 & & \\
\hline & & 97 & 35 a 37 & & \\
\hline & & 98 & 38 a 43 & & \\
\hline & & 99 & 44 a 54 & & \\
\hline
\end{tabular}




\begin{tabular}{|c|c|c|c|c|c|}
\hline \multicolumn{2}{|c|}{ FACTOR NUMÉRICO } & \multicolumn{4}{|c|}{ FACTOR FLUIDEZ VERBAL } \\
\hline Percentil & Puntuación natural & Percentil & Puntuación natural & Percentil & Puntuación natural \\
\hline 4 & 0 & 1 & 0 a 7 & 89 & 50 \\
\hline 7 & 1 & 2 & $8 \mathrm{a} 12$ & 90 & 51 \\
\hline 10 & 2 & 3 & 13 a 14 & 91 & 52 \\
\hline 14 & 3 & 4 & 15 a 17 & 92 & 53 \\
\hline 19 & 4 & 5 & 18 & 94 & 54 a 55 \\
\hline 22 & 5 & 6 & 19 & 95 & 56 \\
\hline 28 & 6 & 7 & 20 & 96 & 57 \\
\hline 33 & 7 & 8 & 21 & 97 & 58 a 59 \\
\hline 38 & 8 & 9 & 22 & 98 & 60 a 62 \\
\hline 44 & 9 & 10 & 23 & 99 & 63 a 75 \\
\hline 51 & 10 & 12 & 24 & & \\
\hline 59 & 11 & 15 & 25 & & \\
\hline 64 & 12 & 16 & 26 & & \\
\hline 71 & 13 & 18 & 27 & & \\
\hline 79 & 14 & 21 & 28 & & \\
\hline 82 & 15 & 23 & 29 & & \\
\hline 86 & 16 & 26 & 30 & & \\
\hline 89 & 17 & 30 & 31 & & \\
\hline 92 & 18 & 33 & 32 & & \\
\hline 94 & 19 & 38 & 33 & & \\
\hline 96 & 20 a 21 & 40 & 34 & & \\
\hline 97 & 22 a 24 & 44 & 35 & & \\
\hline 98 & 25 a 26 & 50 & 36 & & \\
\hline 99 & 27 a 70 & 52 & 37 & & \\
\hline & & 56 & 38 & & \\
\hline & & 60 & 39 & & \\
\hline & & 64 & 40 & & \\
\hline & & 67 & 41 & & \\
\hline & & 69 & 42 & & \\
\hline & & 73 & 43 & & \\
\hline & & 76 & 44 & & \\
\hline & & 79 & 45 & & \\
\hline & & 80 & 46 & & \\
\hline & & 83 & 47 & & \\
\hline & & 85 & 48 & & \\
\hline & & 86 & 49 & & \\
\hline
\end{tabular}

Fuente: Elaboración propia 
Tabla 12. Baremo Percentilar Masculino

\begin{tabular}{|c|c|c|c|c|c|}
\hline \multicolumn{2}{|c|}{ FACTOR VERBAL } & \multicolumn{2}{|c|}{ FACTOR ESPACIAL } & \multicolumn{2}{|c|}{ FACTOR RAZONAMIENTO } \\
\hline Percentil & Puntuación natural & Percentil & Puntuación natural & Percentil & Puntuación natural \\
\hline 1 & 0 a 2 & 19 & 0 a 2 & 1 & 0 \\
\hline 2 & 3 a 5 & 20 & 3 & 3 & 1 \\
\hline 3 & 6 & 21 & 4 & 8 & 2 \\
\hline 4 & 7 & 23 & 5 & 15 & 3 \\
\hline 5 & 8 & 24 & 6 & 24 & 4 \\
\hline 7 & 9 & 25 & 7 & 30 & 5 \\
\hline 9 & 10 & 28 & 8 & 36 & 6 \\
\hline 11 & 11 & 29 & 9 & 43 & 7 \\
\hline 17 & 12 & 30 & 10 & 50 & 8 \\
\hline 24 & 13 & 34 & 11 & 58 & 9 \\
\hline 31 & 14 & 36 & 12 & 63 & 10 \\
\hline 37 & 15 & 37 & 13 & 69 & 11 \\
\hline 45 & 16 & 39 & 14 & 74 & 12 \\
\hline 51 & 17 & 43 & 15 & 80 & 13 \\
\hline 56 & 18 & 46 & 16 & 84 & 14 \\
\hline 61 & 19 & 49 & 17 & 86 & 15 \\
\hline 68 & 20 & 52 & 18 & 89 & 16 \\
\hline 73 & 21 & 55 & 19 & 91 & 17 \\
\hline 77 & 22 & 59 & 20 & 92 & 18 \\
\hline 82 & 23 & 61 & 21 & 94 & 19 \\
\hline 86 & 24 & 64 & 22 & 95 & 20 \\
\hline 90 & 25 & 68 & 23 & 97 & 21 a 23 \\
\hline 92 & 26 & 71 & 24 & 98 & 24 a 35 \\
\hline 94 & 27 a 28 & 75 & 25 & 99 & 40 a 30 \\
\hline 96 & 29 & 77 & 26 & & \\
\hline 97 & 30 & 80 & 27 & & \\
\hline 98 & 31 a 33 & 81 & 28 & & \\
\hline 99 & 34 a 50 & 85 & 29 & & \\
\hline & & 86 & 30 & & \\
\hline & & 88 & 31 & & \\
\hline & & 89 & 32 & & \\
\hline & & 91 & 33 & & \\
\hline & & 92 & 34 & & \\
\hline & & 94 & 35 a 36 & & \\
\hline & & 95 & 37 a 38 & & \\
\hline & & 96 & 39 a 40 & & \\
\hline & & 98 & 41 a 43 & & \\
\hline & & 99 & 44 a 54 & & \\
\hline
\end{tabular}




\begin{tabular}{|c|c|c|c|c|c|}
\hline \multicolumn{2}{|c|}{ FACTOR NUMÉRICO } & \multicolumn{4}{|c|}{ FACTOR FLUIDEZ VERBAL } \\
\hline Percentil & Puntuación natural & Percentil & Puntuación natural & Percentil & Puntuación natural \\
\hline 4 & 0 & 1 & 0 a 13 & 86 & 50 \\
\hline 8 & 1 & 2 & 14 a 16 & 88 & 51 \\
\hline 9 & 2 & 3 & 17 a 18 & 89 & 52 \\
\hline 13 & $\overline{3}$ & 4 & 19 & 91 & 53 \\
\hline 16 & 4 & 5 & 20 & 92 & 54 \\
\hline 19 & 5 & 6 & 21 a 22 & 94 & 55 \\
\hline 24 & 6 & 8 & 23 & 95 & 56 a 57 \\
\hline 27 & 7 & 9 & 24 & 96 & 58 a 59 \\
\hline 33 & 8 & 11 & 25 & 97 & 60 \\
\hline 39 & 9 & 15 & 26 & 98 & 61 a 64 \\
\hline 46 & $\overline{10}$ & 17 & 27 & 99 & 65 a 75 \\
\hline 53 & 11 & 21 & 28 & & \\
\hline 59 & 12 & 24 & 29 & & \\
\hline 64 & 13 & 26 & 30 & & \\
\hline 69 & 14 & 28 & 31 & & \\
\hline 75 & 15 & 32 & 32 & & \\
\hline 79 & 16 & 35 & 33 & & \\
\hline 84 & 17 & 38 & 34 & & \\
\hline 86 & 18 & 42 & 35 & & \\
\hline 89 & 19 & 46 & 36 & & \\
\hline 91 & 20 & 50 & 37 & & \\
\hline 93 & 21 & 53 & 38 & & \\
\hline 95 & 22 & 56 & 39 & & \\
\hline 96 & 23 & 60 & 40 & & \\
\hline 97 & 24 & 63 & 41 & & \\
\hline 98 & 25 a 26 & 66 & 42 & & \\
\hline 99 & 27 a 70 & 70 & 43 & & \\
\hline & & 74 & 44 & & \\
\hline & & 76 & 45 & & \\
\hline & & 78 & 46 & & \\
\hline & & 80 & 47 & & \\
\hline & & 82 & 48 & & \\
\hline & & 84 & 49 & & \\
\hline
\end{tabular}

Fuente: Elaboración propia 


\section{DISCUSIÓN}

Se ha elaborado los datos normativos hondureños de la muestra general para la onceava versión del test de aptitudes mentales primarias, PMA. Fue confirmado el argumento esgrimido en la prueba de hipótesis sobre la existencia de diferencias sexuales en aptitudes mentales por medio de la prueba t para medias independientes con hipótesis direccional, lo que permitió comprobar parcialmente la existencia de esas diferencias por sexo sólo en tres subpruebas: verbal, espacial y numérico; las medias son diferentes.

De acuerdo con lo anterior, Echavarri, Godoy y Olaz (2007) sostienen que los efectos del sexo se encuentran en pruebas de habilidades específicas; así, varias investigaciones han demostrado que los varones consiguen puntajes más altos en tests que implican razonamiento espacial y las mujeres generalmente aventajan a los hombres en habilidades verbales, especialmente en lenguaje.

Dados los resultados anteriores y para permitir al psicólogo comparar el puntaje de un sujeto con el rendimiento del grupo más pertinente, se construyó el baremo por sexo. Al respecto, Wesman (1949) (citado por Casullo y Echeverría, 2010) valora la importancia de reconocer las diferencias entre los géneros al elaborar y estandarizar tests y además para la interpretación de los puntajes.

El análisis de varianza para los promedios de la muestra de tipificación, según la carrera del nivel medio, permite aceptar parcialmente la hipótesis alterna, en el sentido de que existen diferencias significativas en los subtest de comprensión verbal, razonamiento y comprensión numérica. Los sujetos acusaron patrones de puntuaciones diferentes que pueden estar asociados al énfasis temático en la formación académica media, a excepción de las calificaciones obtenidas en las subpruebas de concepción espacial y fluidez verbal.

El tipo de educación (técnica o humanística) influye sobre los puntajes obtenidos en las pruebas de comprensión verbal, razonamiento y números. Los sujetos con una educación humanística muestran un rendimiento más alto en comprensión verbal que los de carreras técnicas, mientras que estos últimos obtuvieron puntajes más altos en el factor espacial y los de modalidad administrativo y comercio mostraron mejores puntuaciones medias en números.

En una investigación que comparó los baremos argentinos y estadounidenses, Stefa- 
ni, Rodríguez y Minzi (1976) estudiaron los cambios de los puntajes de los sujetos con educación técnica con relación a los ocurridos en los sujetos con educación humanística; concluyeron que difieren significativamente en los factores: espacial y el numérico. De este modo, se considera que la incidencia de la educación en el desempeño de test de habilidades hace que un baremo extranjero sea poco adecuado para evaluar a los hondureños. Además, hay una tendencia en la muestra de tipificación a acusar en el PMA calificaciones distintas según el rango de edad; así, las calificaciones medias fueron diferentes en los factores espacial, numérico y razonamiento de acuerdo al rango de edad.

Anastasi y Urbina (1998) plantean que las últimas investigaciones sobre habilidades mentales en todos los niveles de edad revelan considerables diferencias individuales, de tal manera que estas -en cualquier nivel de edad- son mayores que las diferencias promedios entre los mismos grupos de edad, por lo que se evidencia un importante traslape en las distribuciones de las puntuaciones obtenidas por los sujetos en edades distintas en pruebas de aptitudes mentales.

Las autoras de esta investigación mencionan estudios que sugieren que los cambios culturales y factores experienciales, además de un ambiente favorable, oportunidades variadas de estimulación intelectual y mantenimiento de un estilo flexible de vida, más que la edad son los que producen diferencias en las calificaciones de pruebas de habilidades. Por tanto, los resultados del PMA pueden entenderse mejor dentro de un marco contextual.

De acuerdo con lo planteado anteriormente, se procedió a la comparación de los datos normativos por centro regional. Tal como lo expresan Anastasi y Urbina (1998), se observó que en todos los subtest que mide el PMA existen diferencias significativas, ya que el contexto es determinante en la forma en que los sujetos responden.

Es importante recalcar que los psicómetras tienden a realizar baremos de instrumentos psicométricos para poblaciones generales, pero resulta evidente y se reconfirma la necesidad de establecer baremos más específicos para no se penalizar a quienes por diferencias culturales, educativas y socioeconómicas, rinden de manera diferente con independencia de su pertenencia a una misma nación. Por ejemplo, Aragón y Silva (2004) recomiendan ser cuidadosos cuando se aplican pruebas baremadas en una población ajena, aunque se piense que son convenientes por la similitud del idioma.

También se compararon las calificaciones de los sujetos según los baremos españoles y hondureños con el cálculo del coeficiente producto-momento de Pearson. 
Camacho (2008) explica que este es un índice que mide el grado de covariación entre distintas variables relacionadas linealmente. Asimismo, Coolican (2005) señala que la correlación de Pearson se basa en la idea de la dispersión de desviaciones de la media en cada grupo de datos.

Todos los coeficientes encontrados oscilan entre 0.7 y 0.9 , excepto en el factor espacial que obtuvo una correlación positiva, pero moderada de 0.48 . De este modo, se evidencian diferencias significativas entre los percentiles obtenidos por los participantes según los baremos españoles y los hondureños, lo que confirma la necesidad de construir instrumentos que midan atributos psicológicos con equidad y pertinencia para la óptima toma de decisiones en los diferentes campos de la intervención psicológica.

Se observó que las puntuaciones según el baremo español son más altas que la de los hondureños, en especial en los factores: espacial y razonamiento; sin embargo, en el factor numérico estas diferencias son mínimas ( $r=0.48)$. Resultados similares se encontraron cuando se compararon los baremos de niños mexicanos con los de niños españoles, los investigadores también observaron que la dispersión de los datos alrededor de los puntajes medios es mayor para la población mexicana comparada con la española (Aragón, y Silva, 2004).

\section{CONCLUSIONES}

En la Universidad Nacional Autónoma de Honduras, la onceava edición del test de aptitudes mentales primarias de Thurstone reflejó una distribución normal en los cinco factores mentales medidos, con lo que se demostró su poder discriminativo, ya que se separó a aquellos sujetos que obtienen puntuaciones altas, medias o bajas; lo que confirma la hipótesis principal que se planteó en el presente estudio.

Al comparar los resultados obtenidos por los participantes de acuerdo al sexo, se hacen evidentes las diferencias significativas en las medias de puntuaciones de las subpruebas de comprensión verbal, espacial y numérica. Los promedios de las puntuaciones en el factor espacial y en el numérico son significativamente más altos en los varones; en el factor de comprensión verbal el promedio es más alto en las mujeres.

Cuando se compararon los puntajes obtenidos por los sujetos de la muestra con 
relación a la carrera cursada en educación media, se observó que en los factores de comprensión verbal, razonamiento y numérico se suscitaron diferencias estadísticamente significativas. La media de las puntuaciones en el factor de comprensión verbal y en razonamiento, fueron más altos en los que estudiaron el bachillerato en ciencias y letras y el actual científico humanista; en el factor numérico los promedios fueron significativamente más altos en los que estudiaron carreras de la modalidad administrativa y comercio. Esto confirma el efecto de la educación en el rendimiento de esta prueba psicométrica.

Para los factores espacial, razonamiento y numérico, se acusaron diferencias significativas con relación a la edad de los participantes. Las medias más altas en concepción espacial y razonamiento las obtuvieron los sujetos de 18 a 22 años. Para el factor de comprensión numérica la obtuvieron los estudiantes con edades entre 28 a 32 años, lo que posiblemente esté influenciado por la experiencia obtenida en el contexto laboral.

En cuanto a la comparación de medias de las puntuaciones tipificadas de acuerdo al centro regional, se observó que sus medias son diferentes en todos los factores medidos. Los promedios de las puntuaciones fueron mucho más altos en los participantes de la ciudad universitaria de Tegucigalpa.

La correlación entre las puntuaciones obtenidas por los sujetos del presente estudio, según el baremo español, es significativamente más baja que cuando se convirtieron los puntajes directos al baremo que se construyó y se presenta en este informe, confirmando así la necesidad de contar con datos normativos justos que coadyuven a la toma de decisiones con equidad y pertinencia.

\section{RECOMENDACIONES}

Es conveniente determinar la confiabilidad de las subpruebas mediante procedimientos estadísticos propios de las pruebas de velocidad, ya que por la naturaleza del estudio no se realizó. La intención no era comparar culturalmente las aptitudes mentales de los sujetos españoles versus los hondureños, pero las diferencias encontradas sirven para fortalecer el objetivo general de este estudio. En consecuencia, siempre se debe ser cuidadosos cuando se aplican pruebas baremadas en una población que no es la local, aun pensando, como ocurre muchas veces, que se tienen similitudes por hablar el mismo idioma. 
Dadas las diferencias significativas por centro regional, edad y modalidad de educación media, se requiere la elaboración de datos normativos de acuerdo a estos elementos. Este sería un excelente complemento de la presente investigación, ya que se pueden utilizar los mismos datos recolectados y la tabla de datos normativos para la muestra general.

\section{AGRADECIMIENTOS}

A los estudiantes de la Carrera de Psicología que han colaborado con la recolección de datos y a Alfonso Escobar Ney, por sus aportes. Gracias también a Miguel Landa Blanco por la traducción del abstract y a la Dirección de Investigación Científica y Posgrado por el apoyo financiero para que este proyecto.

\section{BIBLIOGRAFÍA}

Aiken, L. (2003). Tests psicológicos y evaluación (onceava ed.). México: Pearson Educación.

Anastasi, A., \& Urbina, S. (1998). Tests psicológicos (Séptima edición ed.). México: Prentice Hall.

Andrade, B. \& Rivadeneira, E. (2006). Estudio estadístico del nivel de desarrollo del pensamiento abstracto, mecánico y verbal en aspirantes a ingresar a la Universidad de Guayaquil, periodo 2003-2004. Tesis, Universidad de Guayaquil, Guayaquil.

Aragón, L. \& Silva, Arturo. (2004). Evaluación psicológica en el área educativa. México: Pax México.

Camacho, J. (Abril-Junio de 2008). Asociación entre variables cuantitativas: análisis de correlación. Acta Médica Costarricense, 50(2), 94-96.

Castaneda Furquim, K. C., Rodríguez Solórzano, B. A. \& Rojas Alfaro, F. d. (2006). Diferencias significativas en las aptitudes mentales primarias con respecto al sexo masculino y femenino en estudiantes que cursan el segundo año de bachillerato general en cinco instituciones privadas del Municipio de Santa Tecla durante el primer semest. Tesis, Universidad Francisco Gavidia, Escuela de Psicología, San Salvador.

Casullo, M., \& Echeverría, H. (2010). Test de aptitudes diferenciales DAT: Manual forma T. Buenos Aires: Paidós. 
Comisión Internacional de Test. (2014). El uso de los tests y otros instrumentos de evaluación en investigación. España.

Coolican, H. (2005). Métodos de investigación y estadística en psicología . México: Edit. El Manual Moderno.

Cruz, L. P. \& Cordovés, B. (1985). Validez y confiabilidad del tes de Habilidades Mentales Primarias de Thurstone en estudiantes de la Universidad Nacional Autónoma de Honduras. Tesis, Universidad Nacional Autónoma de Honduras, Carrera de Psicología, Tegucigalpa.

Downie, R. \& Heath, N. (1986). Métodos estadísticos aplicados. Madrid: Ediciones El Castillo.

Echavarri, M., Godoy, J. \& Olaz, F. (2007). Diferencias de género en habilidades cognitivas y rendimiento académico en estudiantes universitarios. Univ. Psychol., $\mathrm{VI}(2), 319-329$.

Grajeda Montalvo, A. T. (2010). Estudio psicométrico de la versión abreviada del test de matrices progresivas de Raven en alumnos de 4to. y 5to. de secundaria de instituciones educativas estatales del distrito de Chaclacayo - Lima. Tesis, Universidad Nacional Mayor de San Marcos, Facultad de Psicología, Lima.

Hernández Sampieri, R., Fernández Collado, C. \& Baptista Lucio, P. (2010). Metodología de la investigación (Quinta ed.). México: Mc Graw Hill.

Lanza, M. (2008). Primer Portal Educativo de Honduras. Recuperado el 15 de julio de 2015, de http://www.portaleducativo.hn/profesores/estructura_sistema_educativo.htm

Matamoros, D., Moncada, G. \& Rivera, I. (Diciembre de 2014). Uso de las pruebas psicológicas en Honduras por psicólogos. Revista Ciencia y Tecnología(15), 71-93.

Morales, M. L. (2009). Psicometría aplicada (Segunda edición ed.). México: Trillas. Morales, P. (2008). Estadística inferencial: el error típico de la media. Madrid, España: Universidad Pontificia Comillas.

Organización Mundial de la Salud. (2011). Guía de intervención mhGAP para los trastornos mentales, neurológicos y por uso de sustencias en el nivel de atención de la salud no especializada.

Pinto Lavin, L. M., Gallardo Rayo, I., \& Wenk Wehmeyer, E. (1991). Determinación de normas para el PMA de L. L. Thurstone en estudiantes del $4^{\circ}$ año de enseñanza media científico humanista de la región metropolitana. Revista de Psicología, 1(2), 25-42.

Rivera Ceverino, G. M., \& Camarena Meza, J. A. (21 de Junio de 2007). Identificación de patrones de inteligencia múltiple utilizando minería de datos en alumnos de educación secundaria. Tesis, Universidad Mayor de San Marcos, Lima.

Rivera de Donaire, E. L., \& Camoriano de Gaborit, G. (1981). Normalización del test 
de Habilidades Mentales Primarias de Thurstone en estudiantes de la Universidad Nacional Autónoma de Honduras. Tesis, Universidad Nacional Autónoma de Honduras, Carrera de Psicología, Tegucigalpa.

Secretaría de Educación. (2003). Currículo Nacional Básico. Tegucigalpa, Honduras. Stefani, D., Rodríguez, F. N., \& Minzi, M. (1976). Relación entre el tipo de educación, la edad y el rendimiento intelectual. Revista Latinoamericana de Psicología, 449-458.

Tapia Alvarez, J. D., \& Chávez Tapia, D. R. (2011). Stad-Core herramienta estadística modular: Una orientación a la construcción y análisis de tests psicológicos. Tesis, Pontificia Universidad Católica del Perú, Facultad de Ciencias e Ingeniería, Lima. Thurstone, L., \& Thurstone, T. (1980). Test de habilidades mentales primarias. Folleto de aplicación. (W. Woyno, \& R. Oñoro, Trads.) México: Manual Moderno. 